\title{
SOLAR OSCILLATOR STRENGTHS AS A DIAGNOSTIC TOOL
}

\author{
E.A. Gurtovenko ${ }^{1}$, R.I. Kostik ${ }^{1}$ and R.J. Rutten ${ }^{2}$ \\ ${ }^{1}$ Main Astronomical Observatory, Academy of Sciences of the Ukrainian SSR, \\ $252127 \mathrm{Kiev}$, USSR \\ ${ }^{2}$ Sterrekundig Instituut, Postbus 80000, NL-3508 TA, Utrecht, The Nether- \\ lands
}

\begin{abstract}
We briefly review the Kiev program for determining oscillator strengths of Fraunhofer lines from the optical solar spectrum, which has recently resulted in a compilation of solar $g f$-values for 1958 lines from 40 chemical elements (Gurtovenko and Kostik 1989). These $g f$-values were determined empirically by fitting solar lines using standard plane-parallel LTE modelling. Errors in this modelling propagate into the $g f$ values; reversely, the deviations in the latter may serve as diagnostics of the modelling and thus of spectral line formation in the solar photosphere. For a small subset, comparison with reliable laboratory data can be made; for other lines there is information in the differences between fits of the line area and of the line depth.
\end{abstract}

\section{The Kiev oscillator strength program}

The solar photosphere may be regarded as a natural furnace from which Fraunhofer lines originate in order to enable the measurement of their oscillator strengths. As such a furnace, the photosphere provides important advantages: (i) - the number of measurable lines is large; (ii)-the measurable lines are often precisely the ones needed in abundance determinations for other stars; (iii) - the furnace properties are rather well known. In contrast, laboratory measurements used to have very large errors until the precise Oxford measurements became available (Blackwell et al. 1982 and references therein), while the latter are mostly for lines that are less suitable for stellar abundance determinations (see Grotrian diagrams in Rutten and Kostik 1988).

In the early eighties a program was started at Kiev to determine empirical solar oscillator strengths, following the classical example set by Holweger (1967). Its first results were two extensive lists of Fe I $g f$-values (Gurtovenko and Kostik 1981a, 1981b). They were found to be of good quality, both in comparisons with laboratory data (Wiese 1983, Cowley and Corliss 1983) and in studies of their internal consistency (Rutten and Kostik 1982, Rutten and Zwaan 1983, Rutten and Van der Zalm 1984).

The sensitivity of such fits to the choice of atmospheric model and to NLTE effects was analysed by Rutten and Kostik (1982). They resolved the apparent conflict between the quality of these LTE fits and the large departures from LTE derived for Fe I lines by Lites (1972, Athay and Lites 1972). Rutten and Kostik found that the empirical LTE 

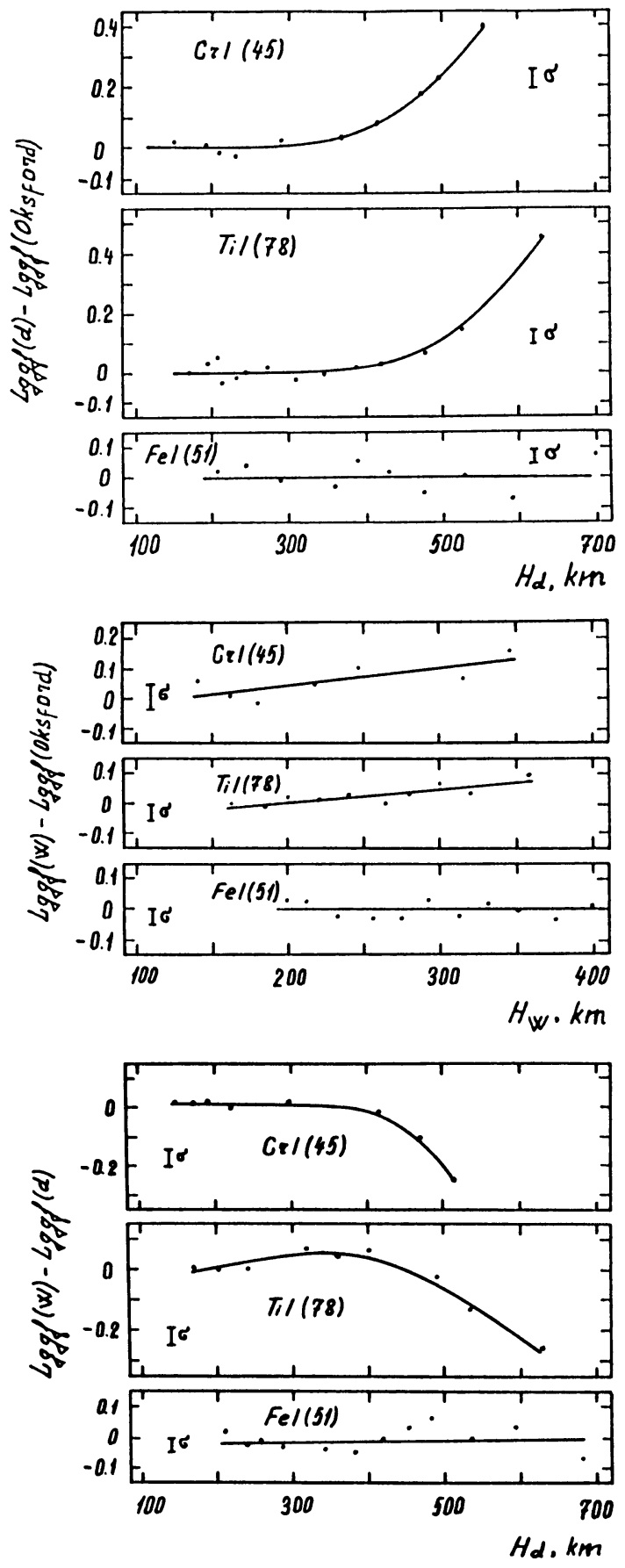

Figure 1. Logarithmic differences between $g f$-values determined by fitting line depths in the Jungfraujoch Atlas of the solar spectrum and laboratory measurements from Oxford.

Figure 2. Logarithmic differences between $g f$-values determined by fitting solar equivalent widths and laboratory measurements from Oxford.

Figure 3. Logarithmic differences between solar $g f$-values from line widths and line depths respectively, for the lines of overlap with Oxford measurements. 
model photosphere of Holweger and Müller (1974) "masks" NLTE departures in iron lines even if the latter are large-as they should be if the solar atmosphere has as cool a temperature minimum as it has in the HSRA and VAL3C standard models (Gingerich et al. 1971, Vernazza et al. 1981). However, recent NLTE modelling with better ultraviolet line blanketing indicates that the upper photosphere is not so cool after all (Avrett 1985, Maltby et al. 1986, Anderson 1989), and that the LTE Holweger-Müller model has actually furnished a correct description of spatially-averaged photospheric line formation all along (Rutten 1988a). This issue is reviewed at length in Rutten (1988b); the point here is that the Kiev fits assuming LTE and the Holweger-Müller model provide accurate $g f$-values for most iron lines whether their actual departures from LTE are large or small. Thus, the solar furnace is an excellent one, without the sensitivity to deviations from LTE that beset earlier laboratory measurements of iron lines using free-burning arcs.

In addition, there are other error sources. The most important ones are unresolved blending and the use of empirical fitting parameters (micro- and macroturbulence, collisional damping enhancement) to correct for the effects of spatial and temporal averaging (Gurtovenko and Sheminova 1986). Their influence on the iron-line fits was tested by Rutten and Kostik (1988), who found that 0.1 dex precision is obtainable for most iron lines. Nordlund (1984) found larger differences when comparing such standard modelling with line profile fits based on his numerical simulation of the solar granulation. However, we expect that such differences will be smaller for his new elastic simulations (Rutten $1988 \mathrm{~b})$. In summary, the Kiev Fe I $g f$-values are generally reliable to within $0.1 \mathrm{dex}$ $(25 \%)$.

\section{New results}

The success described above has led the Kiev workers to continue their effort. A new compilation with solar $g f$-values for 1958 lines from 40 elements has just appeared (Gurtovenko and Kostik 1989). These are obviously useful for stellar abundance determinations, but they may also serve as diagnostics of photospheric line formation. We address the latter usage here. Since the fits are obtained from the comparison of theory and observations, they contain errors due to modelling assumptions of which the characteristics may vary with solar line strength and with atomic parameters. Such errors and their dependences can be employed to test the validity of the standard modelling and to study Fraunhofer line formation adding other spectra than Fe I.

The measured quantity per $\operatorname{line}$ is $\log L=\log (A g f)$ where $\log A=\log \left(N_{e l} / N_{H}\right)+12$. Thus, each measurement error $\delta \log L$ has $\delta \log L=\delta \log g f+\Delta \log A$ where $\Delta \log A$ is a correction to the adopted elemental abundance value which is the same for all lines from that element. The remaining error-per-line $\delta \log g f$ is the one which concerns us here. There are two sets of $g f$ measurements, obtained from fitting equivalent widths and central depths respectively: $g f_{w}$ values and $g f_{d}$ values. There are two ways of studying errors in these: analysing the differences of each set with results from others, and analysing the differences between the two sets. We haven't performed such analysis yet in detail; here, we show first results that are indicative of the information content of the new measurements.

Figures 1 and 2 show the differences between the Kiev results and the Oxford measurements for the $g f_{d}$ and $g f_{w}$ values respectively, against the corresponding height of 


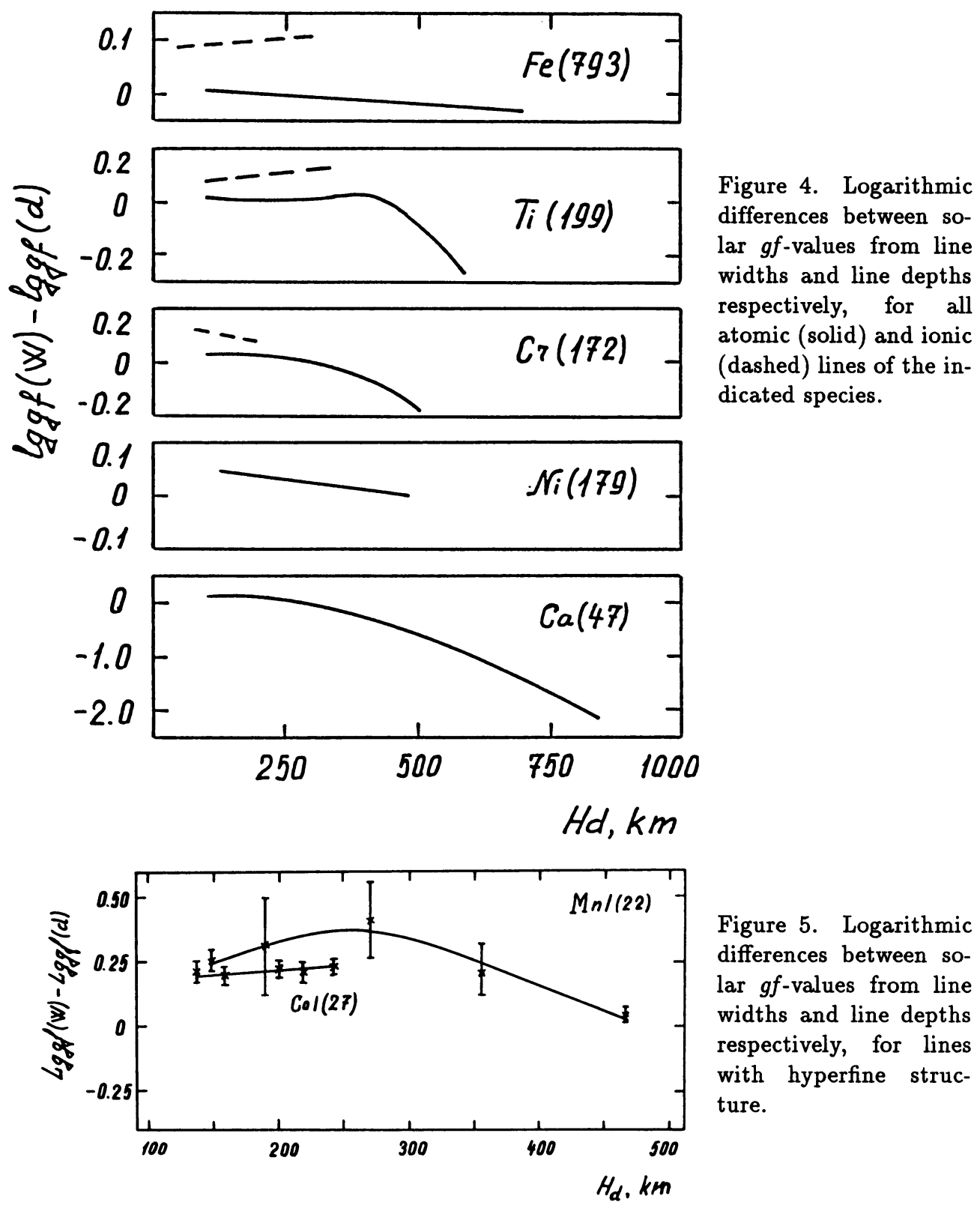


formation in the photosphere. These diagrams contain averages for all lines for which Oxford results are available, from Fe I ( 51 lines), Ti I (78 lines) and $\mathrm{Cr}$ I (45 lines). The bars denote one-sigma standard deviations. The absence of a trend in the Fe $\mathrm{I}$ lines is to be expected because the nonthermal broadening parameters in the modelling were derived by fitting representative Fe I lines using the Oxford $g f$-values and the HolwegerMüller model itself was originally derived from iron lines. The differences are also small for the $\mathrm{Ti} \mathrm{I}$ and $\mathrm{Cr}$ I lines, except for the strongest lines formed in the upper photosphere which have deeper cores in the observed spectrum than the modelling provides for if we assume that the Oxford values are correct.

Figure 3 displays the signature of these trends in a $\log g f_{w}-\log g f_{d}$ plot against height for the same lines. Note the difference in the weak-line parts between the $\mathrm{Cr} I$ and the Ti I results.

Figure 4 shows the composite averages for all lines of iron (793 lines), titanium (199 lines), chromium (172 lines), nickel (179 lines) and calcium (47 lines), respectively from their neutral stages (full lines) and first ionization stages (dashed). Fe I now shows a slight trend, due to the addition of numerous other lines than the Oxford ones. A similar trend is shown by $\mathrm{Ni}$. The stronger lines of $\mathrm{Cr} \mathrm{I}$ and $\mathrm{Ti}$ I display the downturns already evident in the smaller samples discussed above. Ca I shows similar behaviour but with a much larger amplitude. All ions show positive differences. Fe II displays a slight opposite trend, increasing with height.

It is already clear that these features have to do with solar line formation and not with inaccuracies in atomic parameters such as ionization potentials, partition functions etc. (see Grevesse 1984). Also, the trends cannot be removed within the constraints of the standard modelling, i.e. by changing the temperature structure or the turbulence parameters. Their explanation requires at least the introduction of departures from LTE in the excitation or ionization equilibria or both, and possibly of inhomogeneous structure not mimicked through nonthermal broadening.

A final point is that such difference plots may also serve to display hyperfine and isotope structure. These influence spectral line profiles just as microturbulence does; in general, their underestimation results in too large $g f_{w}$ and too small $g f_{d}$ values and in a specific pattern in the difference plots: first an increase and then a decrease (Gurtovenko and Sheminova 1988). Such behaviour is seen in Figure 5 which displays $\log g f_{w}-\log g f_{d}$ differences for Mn I and Co I lines with hyperfine structure.

\section{Conclusion}

Our display of difference plots from the new Kiev compilation shows that there is interesting behaviour pointing to deficiencies in the standard modelling. Although the iron lines and probably also the nickel lines are represented quite well assuming LTE and the Holweger-Müller model, there are significant deviations for the strong lines from other spectra, requiring more detailed analysis. For $\mathrm{Ti} \mathrm{I}$ and $\mathrm{Cr}$ I these deviations may simply display strong-line photon losses in excess of typical Fe I behaviour, but there is no immediate explanation for the very large deviations in $\mathrm{Ca} \mathrm{I}$ and for the splits between atom and ion lines. We conclude that, again, empirical solar $g f$-values may be employed as a diagnostic of line formation in the solar atmosphere. 


\section{References}

Anderson, L. S.: 1989, Astrophys. J. 339, 558

Athay, R. G. and Lites, B. W.: 1972, Astrophys. J. 176, 809

Avrett, E. H.: 1985, in Lites, B. W. (Ed.), Chromospheric Diagnostics and Modeling, p. 67, National Solar Observatory Summer Conference, Sacramento Peak Observatory, Sunspot, New Mexico

Blackwell, D. E., Petford, A. D., Shallis, M. J., and Simmons, G. J.: 1982, Monthly Notices Roy. Astron. Soc. 199, 43

Cowley, C. R. and Corliss, C. H.: 1983, Monthly Notices Roy. Astron. Soc. 203, 651

Gingerich, O., Noyes, R. W., Kalkofen, W., and Cuny, Y.: 1971, Solar Phys. 18, 347

Grevesse, N.: 1984, Physica Scripta T8, 49

Gurtovenko, E. A. and Kostik, R. I.: 1981a, Astron. Astrophys. Suppl. 46, 239

Gurtovenko, E. A. and Kostik, R. I.: 1981b, Astron. Astrophys. Suppl. 47, 193

Gurtovenko, E. A. and Kostik, R. I.: 1989, Fraunhofer Spectrum and the System of Solar Oscillator Strengths, Naukova Dumka, Kiev

Gurtovenko, E. A. and Sheminova, V. A.: 1986, Solar Phys. 106, 237

Gurtovenko, E. A. and Sheminova, V. A.: 1988, Kinematika i Fizika Nebesnich Tel. 4, 18

Holweger, H.: 1967, Zeitschr. f. Astrophysik 65, 365

Holweger, H. and Müller, E. A.: 1974, Solar Phys. 39, 19

Lites, B. W.: 1972, Observation and Analysis of the Solar Neutral Iron Spectrum, NCAR Cooperative Thesis No. 28, High Altitude Observatory, Boulder

Maltby, P., Avrett, E. H., Carlsson, M., Kjeldseth-Moe, O., Kurucz, R. L., and Loeser, R.: 1986, Astrophys. J. 306, 284

Nordlund, A.: 1984, in Keil, S. L. (Ed.), Small-Scale Dynamical Processes in Quiet Stellar Atmospheres, p. 181, National Solar Observatory Summer Conference, Sacramento Peak Observatory, Sunspot, New Mexico

Rutten, R. J.: 1988a, in Cayrel de Strobel, G. and Spite, M. (Eds.), The Impact of Very High $S / N$ Spectroscopy on Stellar Physics, p. 367, IAU Symposium 132, Reidel, Dordrecht

Rutten, R. J.: 1988b, in Viotti, R., Vittone, A., and Friedjung, M. (Eds.), Physics of Formation of FeII Lines Outside LTE, p. 185, IAU Colloquium 94, Reidel, Dordrecht

Rutten, R. J. and Kostik, R. I.: 1982, Astron. Astrophys. 115, 104

Rutten, R. J. and Kostik, R. I.: 1988, in Viotti, R., Vittone, A., and Friedjung, M. (Eds.), Physics of Formation of FeII Lines Outside LTE, p. 83, IAU Colloquium 94, Reidel, Dordrecht

Rutten, R. J. and van der Zalm, E. B. J.: 1984, Astron. Astrophys. Suppl. 55, 143

Rutten, R. J. and Zwaan, C.: 1983, Astron. Astrophys. 117, 21

Vernazza, J. E., Avrett, E. H., and Loeser, R.: 1981, Astrophys. J. Suppl. Ser. 45, 635

Wiese, W. L.: 1983, in West, R. M. (Ed), Highlights of Astronomy, 6, 795 